\title{
Introduction
}

\section{Space, the vestibular system, and MIT's Man-Vehicle Laboratory}

\author{
Laurence R. Young \\ Department of Aeronautics and Astronautics, Massachusetts Institute of Technology, Cambridge, MA, USA \\ Tel.: +1 617253 7759; E-mail: lry@mit.edu
}

Received 23 November 2009

Accepted 5 May 2010

On March 5, 2009, a symposium on Strategic Planning Activity on Aerospace Medical and Human Factors Challenges was held at the Man-Vehicle Laboratory (MVL) at the Massachusetts Institute of Technology. The program was divided into three sections: Aviation Human Factors, Sensorimotor Challenges in Space, and Earth and Clinical Applications. The symposium was held on the occasion of the 65th birthday of Dr. Charles M. Oman, the Director of the MVL; participants and MVL colleagues were invited to submit manuscripts for this special issue.

The Man-Vehicle Laboratory was founded in 1962, with a goal of better defining the physiological and cognitive limitations of pilots and passengers of aircraft and spacecraft, and to optimize overall human-vehicle system effectiveness and safety. As part of this mission, providing a better understanding of the role that the vestibular system plays in motor and sensory processing, particularly during the skilled tasks involved in vehicular flight, has been an important part of the MVL mission (http://mvl.mit.edu/).

The MVL has long had a close collaboration with NASA, initially to better model and understand the phenomenon of "Space Motion Sickness" (otherwise called "Space Adaptation Syndrome"). From its original emphasis on manual control and the role of motion in determining pilot reactions, MVL's interest evolved to a broader concentration on posture, biomechanics, eye movements, motion sickness and the diagnosis of vestibular disorders, applying engineering techniques to attempt to better understand vestibular physiology, particularly in unusual environments. Experiments have been conducted in airplanes and in space as well as in the clinic and the laboratory. From the first measurements of vestibular adaptation to weightlessness to current emphasis on sensory-motor disturbances, studies have examined human perceptual, oculomotor and postural reactions to unusual gravito-inertial environments. Experimental sites have included over 10 space shuttle flights, the Russian MIR station and on the International Space Station. MVL investigators have also actively pursued mathematical modeling of sensorimotor processes, particularly the issue of adaptation of systems to altered environments.

The papers in this Special Issue exemplify the broad scope of problems and approaches that characterize the MVL and the interests of Dr. Oman. The first paper, from Shinder and Taube, examines the neural basis of vestibular inputs to higher cortical centers that can provide insights into regions of the brain involved in self-motion awareness, heading and orientation, and the differentiation of "self" from the (usually-stationary) surroundings.

In orbital flight, signals from the vestibular system continue to signal head rotations, but no longer signal "orientation with respect to gravity". One sense that may "substitute" for orientation cues in space is the visual system, allowing the subject to orient with respect 
to the static walls, floor, ceiling, and other fixed aspects of the immediate environment. Experiments by Harris and his colleagues examine the ability of the visual system on earth to maintain accurate and stable perception of orientation when the visual scene is manipulated as though the subject were moving or changing orientation, but in the absence of actual motion.

The unusual "micro-gravity" environment of orbital and sub-orbital flight frequently leads to a period of reduced human performance as a result of motion sickness. Karmali and Shelhamer investigate strategies for pre-adapting subjects to these environments, with particular attention to the differing demands of short, sub-orbital flights and more extended orbital missions (where adaptation might be factored into the initial days of the mission).

While we do not have an extended earth-based "micro-gravity" environment (other than a brief period during parabolic flight, followed by a period of "hypergravity"), we can alter the normal $1 \mathrm{~g}$ "vertical reference" frame using prolonged horizontal bed rest. Similarly, in orbital flight, centrifugation can be employed to produce a body-vertical acceleration. Experiments by Jarchow and Young have examined the combination of bed rest and periods of centrifugation to examine how the perception of vertical was influenced by three weeks of bed rest, with half of the subjects then subjected to feet-out centrifugation (producing a $1 \mathrm{~g}$ centripetal acceleration at the heart).

How does microgravity affect cognition? Grabherr and Mast review the literature on cognitive abilities of subjects in parabolic and orbital flights, focusing on mental imagery, particularly as it applies to the ability to maintain an appropriate representation of the spatial environment and the subject's orientation in this environment. Having a better understanding of the effect of microgravity on these cognitive processes may suggest forms of mental training that could minimize any adverse effects.

Two presentations emphasized the multidisciplinary approach that the MVL brings to an understanding of the vestibular system for patients with vestibular deficits. Wall and Kentala use a vestibular prosthesis consisting of a linear accelerometer and rate gyroscope mounted on the subject's back, with vibrators on the subject's stomach and back. They explore how altering the feedback signals from the sensors can assist vestibulopathic subjects in maintaining an upright standing posture with minimal sway. The final paper by Dan Merfeld and his colleagues explores several challenges facing clinicians attempting to diagnose and treat vestibular disorders. One is the variable sensitivity and specificity of vestibular testing - many patients with obvious deficits can manifest normal caloric and rotatory tests. They review several approaches involving perception, including measuring vestibular thresholds to both rotatory and linear motion. They also review the possibility of developing a vestibular prosthesis, with particular emphasis on techniques for directly stimulating the vestibular nerve.

All of these contributions involve a combination of biological and engineering approaches to understanding the vestibular system and its role in orientation and motor control, all significant themes of the Man-Vehicle Laboratory and the career of its director, Charles Oman. He was one of the first graduate students at the MVL, coming with knowledge of control theory, an interest in biology, and a love of flying, complete with pilot's license. His Master's thesis was on modeling vestibular adaptation to prolonged rotation, while his $\mathrm{PhD}$ investigated the lateral line organ in aquatic animals. He joined the MIT faculty and in 1992 was named the Director of the MVL. His scientific and engineering specialization covers aerospace physiology and human factors, vestibular physiology, human spatial orientation and navigation, motion sickness, flight simulation and virtual environment technology. He has been closely associated with space flight experiments in the Spacelab program - from the very first SL-1 in 1983 through the very last Neurolab mission in 1998, and was co-I or PI on six Spacelabs and a planned Space Station experiment.

In 2003 and 2007, Dr. Oman helped organize two NASA-sponsored international Symposia on the Role of the Vestibular Organs in the Exploration of Space. Proceedings from these Symposia have been published in the Journal of Vestibular Research (Volume 13, Numbers $4 / 5$ and Volume 17, Numbers 5/6), as well as a special issue from neurovestibular adaptation studies supported by the National Space Biomedical Research Institute (Volume 12, Numbers 5/6).

The symposium was supported by the National Space Biomedical Research Institute (NRC AO00005), the Department of Aeronautics and Astronautics at MIT, the Jenks Vestibular Laboratory of the Massachusetts Eye and Ear Infirmary and the Department of Otology and Laryngology of Harvard Medical School. 